\title{
Incidencia de las actitudes cognitivas y afectivas de los consumidores en la defensa de una marca local
}

\section{Incidence of cognitive and affective attitudes of consumers in the defense of a local brand}

María Cristina Otero-Gómez ${ }^{1}$
Wilson Giraldo-Pérez

Recibido: septiembre 12 de 2019

Aceptado: diciembre 07 de 2019

\section{Resumen}

Desde el marketing, este estudio investiga las percepciones que tienen los consumidores sobre la marca local La Catira Industria Láctea, ubicada en el departamento del Meta, Colombia. Tiene como objetivo analizar las actitudes cognitivas y afectivas del consumidor en relación con la marca, con el fin de establecer cuál variable tiene mayor incidencia en su defensa. La recolección de los datos se realizó por medio de 187 encuestas físicas y en línea. A pesar de ser una marca representativa en la región, en el componente afectivo el $27,8 \%$ afirma amar esta marca, valor que no superó los porcentajes para el componente cognitivo, donde el $66,4 \%$ indica que los productos de la marca son de muy buena calidad. Los resultados sugieren que la calidad percibida, es la variable de la actitud cognitiva que más se relaciona con la defensa de la marca. Además, se evidencia la debilidad en el vínculo emocional desde la perspectiva del consumidor, en relación con la marca estudiada.

Palabras clave: marketing, actitud cognitiva, actitud afectiva, defensa de la marca.

\begin{abstract}
From marketing, this study explores the perceptions of consumers about the local brand La Catira Industria Láctea, located in the department of Meta, Colombia. It aims to analyze the cognitive and affective attitudes of the consumer in relation to the brand in order to establish the variable with the greatest impact on its defense. Data collection was carried out through 187 in-person and online surveys. Despite being a representative brand in the region, in the affective component $27.8 \%$ claim to love this brand, a value that did not exceed the percentages for the cognitive component, which shows that $66.4 \%$ claim that the products of the brand are of a very good quality. The results suggest that the perceived quality is the variable of the cognitive attitude that is most related to the defense of the brand. In addition, the weakness in the emotional bond is evidenced from the perspective of the consumer in relation to the brand studied.
\end{abstract}

Keywords: marketing, cognitive attitude, affective attitude, brand defense.

1 Profesional en comercio internacional, Estudiante de Doctorado en Marketing, Universidad de los Llanos, Villavicencio, Colombia. E-mail: motero@unillanos.edu.co. ORCID: 0000-0002-3241-7877

2 Administrador de Empresas, Estudiante de Doctorado en marketing, Universidad de los Llanos, Villavicencio, Colombia. E-mail: wgiraldo@unillanos.edu.co. ORCID: 0000-0001-8514-9805 


\section{Introducción}

A medida que avanzan los procesos de globalización, los consumidores tienen mayores opciones para elegir productos y marcas que se ajusten a sus necesidades; en consecuencia, las empresas regionales se enfrentan a una feroz competencia para atraer y retener a sus clientes. Sumado a esto, existen otros factores externos que dificultan la sostenibilidad de las empresas, como es el caso de la infraestructura vial, que permite el tránsito no solo de mercancías sino de personas tanto residentes como foráneas. Estas dos vertientes son clave para el desarrollo de la presente investigación, la cual tiene como objetivo analizar las actitudes cognitivas y afectivas del consumidor, con el fin de establecer cuál variable tiene mayor incidencia en la actitud conductual, manifestada a través de la defensa de la marca La Catira Industria Láctea (en adelante La Catira).

La empresa a la que pertenece la marca fue fundada en el año 1982 y actualmente se encuentra ubicada en el municipio de Restrepo (M), sobre la ruta turística denominada "El embrujo llanero", por lo que gran parte de su demanda se concentra principalmente en los visitantes procedentes de Villavicencio, Bogotá y otras zonas del centro del país. La Catira cuenta con un amplio portafolio de productos derivados de la leche como son quesos, postres, kumis, yogures, amasijos y tortas, entre otros. Todos sus productos se distribuyen en el punto de fábrica; pero en el caso de los yogures y quesos, cuenta con presencia en supermercados del orden local y nacional, bien sea a través de su propia marca o mediante operaciones de maquila.

Aunque se trata de una empresa con trayectoria en el mercado, también se ve expuesta a situaciones adversas del entorno. La crisis actual derivada del cierre de la vía terrestre que conecta a Bogotá con la Orinoquia colombiana, ha generado múltiples problemas entre los que se cuenta la baja afluencia de turistas y el impacto en los procesos de distribución de la leche. Según cifras de la Federación Nacional de Comerciantes (FENALCO,
2019), por cada día de cierre se pierden del 90 al 95 por ciento de las reservas hoteleras. Por su parte, la Asociación de Lecheros de Mesetas - Meta afirma que "Nosotros le compramos la leche a los campesinos y sacamos el producto del pueblo. Con el último cierre fueron días difíciles, porque nos tocó guardar la leche y esperar a que reabrieran la carretera" (Semana Rural, 2018). Actualmente, el cierre de la vía se ha prolongado durante meses y sin una fecha exacta sobre su apertura, lo que obliga al aparato productivo y al tejido empresarial del Meta a tomar medidas que contrarresten los efectos negativos causados por los factores externos.

Esta amenaza podría convertirse en una oportunidad para trasladar el consumo de productos lácteos que inicialmente estaba en manos del turista o del foráneo, y conducirlo hacia la demanda generada por el residente. En el marketing de relaciones, el fortalecimiento de los lazos entre el consumidor y las marcas son fundamentales para alcanzar una ventaja competitiva sostenible a largo plazo (Cavazos \& Giuliani, 2008); en algunos casos los acercamientos permiten que los clientes se conviertan en defensores, promotores y embajadores de sus marcas favoritas o marcas amadas (Burnasheva, Suh \& Villalobos, 2019). Aunque siempre está presente la variable precio en el momento de la compra, surge como interrogante si la época de crisis hace que los residentes modifiquen sus hábitos de consumo, de tal forma que se privilegie o defienda la producción local. Es posible que existan otras relaciones que conllevan a la defensa de la marca; sin embargo, a partir de la revisión teórica, esta investigación es innovadora en el sentido de establecer una relación empírica entre las actitudes cognitivas reflejadas en el valor de marca, junto con las actitudes afectivas representadas en el amor a la marca, las cuales conllevan a las actitudes conductuales que se manifiestan en los comentarios positivos, persuasión a otros consumidores y en general, en la defensa de la marca. 
Este manuscrito está organizado de la siguiente manera: la primera sección indica el marco teórico que soporta las hipótesis planteadas, junto con la explicación de los métodos utilizados para examinar las relaciones entre las variables. A continuación se presentan los resultados de la parte empírica del estudio, seguido de las discusiones y las conclusiones.

\section{Marco teórico y metodología}

\subsection{Fundamento teórico e hipótesis de la investigación}

En la investigación del marketing, diversos autores se han enfocado en profundizar sobre la importancia de las actitudes de los individuos en relación con una marca (Keller, 1993; Daems, De Pelsmacker \& Moons, 2019). Las actitudes hacia la marca reflejan las percepciones de los consumidores sobre la calidad, la satisfacción y el valor de la marca (VM), además de predecir comportamientos posteriores como la búsqueda y la evaluación (Dotson, Fan, Feit, Oldham \& Yeh, 2017; Du, Joo \& Wilbur, 2019). Los estudios sobre la actitud se han desarrollado con el fin de analizar la fortaleza de la marca en relación con la competencia, la evaluación del rendimiento y otras tácticas de marketing, en aras de proporcionar un indicador temprano de ventas futuras, por lo que a los encuestados se les pide que respondan una variedad de preguntas relacionadas con la notoriedad, la calidad (Dotson et al., 2017), las asociaciones con la marca y la lealtad (Keller, 1993). Estas variables conforman el VM, entendido como la percepción de los consumidores en cuanto a superioridad general de la marca en comparación con otras marcas (Lassar, Mittal \& Sharma, 1995); lo integran: la calidad percibida (CP), la notoriedad (N), las asociaciones (AS) y la lealtad (L) (Aaker, 1996).

Al confrontar el enfoque anterior con la literatura empírica, se ha encontrado que las actitudes hacia la marca pueden estar relacionadas con la CP del consumidor, a partir de sus creencias sobre los atributos relacionados con el producto y los beneficios funcionales y experimentales (Zeithaml, 1988). Asimismo, Keller (1993), sugiere que $N$ desempeña un papel importante en las actitudes, puesto que se relaciona con el recuerdo y el reconocimiento de la marca por parte del consumidor. Desde la visión de Sürücü, Öztürk, Okumus \& Bilgihan (2019), en las AS se crean actitudes generales de la marca que conllevan a la formación de imágenes y atributos relacionados o no con el producto como son: "la compañía, la persona, el lugar o el evento que conforma la asociación de marca" (Keller, 1993). Igualmente, Keller afirma que la "lealtad ocurre cuando las creencias y actitudes favorables para la marca se manifiestan en el comportamiento de compra repetido" (1993); en tal caso, L puede ser conductual cuando los consumidores compran repetidamente, pero también puede ser actitudinal cuando representan las creencias y las intenciones del consumidor hacia la marca (Gill \& Dawra, 2010). Para este estudio se considera la lealtad actitudinal, puesto que las preguntas del cuestionario apuntan a las valoraciones de las percepciones del consumidor en torno $a \mathrm{~L}$.

Según Morales (1.999), "a través de tres componentes se manifiesta la actitud, ellos son: el cognitivo, el afectivo y el conductual (conativo)". A nivel comercial, en el primero los consumidores adquieren conocimiento sobre un producto o una marca y lo recuerdan; en el segundo desarrollan un afecto positivo o negativo, y en el tercero, los consumidores actúan sobre la base de su conocimiento (Daems et al., 2019). Visto de esta forma, el presente estudio aborda un primer escenario que relaciona las variables $C P, N, A S$ y $L$ con las actitudes cognitivas, puesto que los consumidores tienen una percepción sobre la calidad de un producto, reconocen el nombre de la marca frente a los competidores, le atribuyen una serie de asociaciones y se sienten identificados como consumidores leales (Villarejo, 2002). Sumado a esto, es necesario recordar que las actitudes hacia la marca también se relacionan con el afecto. En consecuencia, se incluye como segundo escenario, la variable amor a la marca (AM) y sus posibles efectos en la defensa de la marca (DM). 
El amor por las marcas es la conexión más intensa emocionalmente entre los consumidores y sus marcas (Schmid \& Huber, 2019). En esta línea, se han analizado: el sentido de apego, el deseo de utilizar la marca, el compromiso de usar la marca en el largo plazo, y el dolor emocional si la marca amada desapareciera, entre otros aspectos (Batra, Ahuvia \& Bagozzi, 2012; Bagozzi, Batra \& Ahuvia, 2017). Aunque $A M$ ha sido ampliamente analizado para los productos hedónicos, también se ha demostrado sus resultados en bienes de consumo utilitario. AM puede utilizarse para predecir las tendencias relacionadas con la marca a mediano y largo plazo y para evaluar el potencial de la marca de manera que no se vea afectado por el tamaño de la empresa (Zarantonello, Formisano \& Grappi, 2016), como es el caso de la empresa objeto de análisis.

Continuando con la clasificación de las actitudes a nivel comercial, desde la perspectiva conductual, los consumidores concretan su conocimiento a través de las acciones (Daems, et al., 2019). Bajo este argumento, la defensa de la marca es el tercer escenario analizado en este estudio, puesto que a partir de los juicios racionales y afectivos, se espera que el consumidor implemente acciones vinculadas a la marca. Walz \& Celuch (2010), definen DM como la promoción o defensa de una organización, producto o marca, por parte de un consumidor a otro. Burnasheva, et al. (2019), en su estudio sugieren la existencia de la relación positiva entre AM y DM; estos autores aseguran que DM es una actitud conductual, toda vez que la primera respuesta de un consumidor es expresar sus reacciones positivas hacia una marca a través de la compra, junto con la comunicación de los aspectos positivos de la marca en entornos específicos. En algunos casos, los defensores de la marca como resultado de su vínculo emocional, ignoran aspectos insatisfactorios con respecto a esta, y tienden a defenderla de las críticas donde "la defensa también incluye el perdón por las malas acciones" (Wallace, Buil \& De Chernatony, 2014).
Conforme a lo anterior, este documento demuestra un interés particular en las percepciones que pueden influir en las respuestas cognitivas, afectivas y conductuales de los consumidores hacia las marcas; por tanto, se sugiere el planteamiento de las siguientes hipótesis:

H1: Las actitudes cognitivas del consumidor contenidas en VM conllevan a la actitud conductual representada en DM.

H2: Las actitudes afectivas del consumidor contenidas en AM conllevan a la actitud conductual representada en DM.

\subsection{Metodología}

\section{Población y muestra}

La muestra está compuesta por individuos residentes principalmente en Villavicencio (M), con el $84 \%$; el $14 \%$ son a habitantes de Restrepo y Cumaral $(\mathrm{M}) ;$ y el $2 \%$ son personas de fuera del departamento del Meta. El único requisito previo para participar en este estudio es que hubieran comprado productos de la marca La Catira durante el último año. La edad de los encuestados se distribuyen de la siguiente forma: entre 18 y 25 años corresponde al 35\%; entre 26 y 35 años con el $16 \%$; entre 36 y 50 años equivalentes al $26 \%$ y mayores de 50 años con el $23 \%$. Se contactaron 204 personas, de las cuales 187 completaron el cuestionario en su totalidad.

En cuanto al perfil de la muestra, el $45 \%$ son hombres y el $55 \%$ mujeres. Alrededor del $45 \%$ realiza compras de forma quincenal y el $30 \%$ mensual. En relación con el nivel de educación, el $45 \%$ ha finalizado sus estudios universitarios y el $45 \%$ son bachilleres. En lo que respecta al estado civil, el $55 \%$ son solteros; le siguen los casados con el $36 \%$ y por último los separados con un $9 \%$. La mayoría de los encuestados son trabajadores dependientes representados en el 36\%, estudiantes $28 \%$ y los independientes $25 \%$. A cerca 
de los ingresos familiares, se pudo identificar que tomando como referencia COP 1.000 .000 el $45 \%$ no supera este promedio, el $36 \%$ está por encima y el $19 \%$ muy por encima.

En cuanto a la medición de las actitudes cognitivas, afectivas y conductuales, se pidió a los encuestados que seleccionaran la opción que mejor representaba sus percepciones. La medición utilizada en este cuestionario, se basó en una escala Likert de cinco puntos que va desde "Muy en desacuerdo" hasta "Muy de acuerdo". Se recopilaron, tradujeron y adaptaron medidas para evaluar cada variable de investigación, a partir de los estudios de Lassar, et al. (1995); Aaker (1996); Yoo, Donthu \& Lee (2000); Netemeyer et al. (2004); Pappu, Quester \& Cooksey (2005); Carroll \& Ahuvia (2006) y Melancon, Noble \& Noble (2011). El instrumento utilizado se presenta en el anexo 1.

\section{Datos y procesamiento}

Para comprobar las hipótesis de la investigación, se recopilaron datos basados en un cuestionario estructurado. El $50 \%$ de los cuestionarios se respondieron de forma física y el 50\% restante se realizó en línea. Las personas fueron contactadas mediante el método de muestreo de bola de nieve no discriminatorio exponencial.
En el procesamiento, se utilizó el software SmartPLS 3, con el cual se realizaron las pruebas de fiabilidad y validez, tratando las variables AM y DM como unidimensionales reflectivas, y la variable VM como multidimensional de segundo orden reflectivo - reflectivo, como se aprecia en los anexos 2 y 3 . Una vez constatado el cumplimiento de los criterios universalmente aceptados para esta verificación, se procedió, mediante el análisis estructural, a comprobar las hipótesis planteadas utilizando el coeficiente de correlación de (rho) Spearman, con el fin de verificar el nivel de asociación entre cada una de las variables independientes con la variable dependiente (DM).

\section{Resultados y discusión}

Un primer elemento considerado en el análisis de los resultados, fue el nivel de asociación que presentaban las variables independientes VM y AM, con la variable dependiente DM. Para lograrlo, se calculó el indicador de correlación de Spearman (rho), el cual arrojó un valor superior para la relación entre VM y DM en comparación con la relación entre AM y DM, pese a que ambos indicadores son significativos estadísticamente, tal como se aprecia en la tabla 1.

Tabla 1. Matriz de correlaciones.

\begin{tabular}{|c|c|c|c|}
\hline & & Actitud cognitiva (VM) & Actitud afectiva (AM) \\
\hline Actitud conductual (DM) & Spearman's rho & $0,783 * * *$ & $0,640 * * *$ \\
\hline
\end{tabular}

El anterior resultado evidencia un comportamiento de DM basado en cogniciones más que en elementos afectivos. Si bien La Catira es una marca con amplia orientación hacia el turista, se esperaba que en épocas de crisis los consumidores de la región manifestaran alguna inclinación por defender o promocionar la marca, pero los resultados demuestran un comportamiento más racional que emocional.
De hecho, cuando se analiza concretamente el ítem "amo esta marca" de la variable AM, los resultados reflejan que del total de las personas encuestadas el $21,4 \%$ se encuentra muy en desacuerdo con esta afirmación y el 20,3\% está en desacuerdo; lo que significa que un $41,7 \%$ de los encuestados no ama esta marca. Estos datos contrastan con el ítem: "Ios productos de La Catira tienen excelentes características" de la variable VM, donde se observa que el $64,7 \%$ de las personas se 
encuentran en los niveles de acuerdo y muy de acuerdo con esta afirmación, siendo coherentes estos resultados con el nivel de asociación de las variables del modelo. Como se observa, existe un débil vínculo emocional entre la marca local y los pobladores, no obstante, la investigación revela una alta valoración en la calidad de los productos. Al mostrar las relaciones que contrastan en los resultados, se amplía la discusión plasmada en los trabajos académicos que previamente analizaron estas variables. Este documento, difiere de los hallazgos presentados por Zarantonello, Formisano y Grappi (2016), quienes descubrieron que AM se relaciona positivamente con las marcas pequeñas; por el contrario, es consistente con la literatura que destaca la alta valoración de una marca en términos de calidad pero que no todos los consumidores satisfechos sienten amor por la marca (Roy, Eshghi \& Sarkar, 2013).

La tabla 2 presenta los resultados del análisis estructural, lo que demuestra la confirmación empírica de las hipótesis propuestas en este manuscrito. De acuerdo con los valores obtenidos, se acepta que tanto las actitudes cognitivas del consumidor contenidas en VM, como las actitudes afectivas del consumidor contenidas en AM, conllevan a la actitud conductual representada en la DM.

Tabla 2. Comprobación de hipótesis.

\begin{tabular}{lllll}
\hline \multicolumn{1}{c}{ Hipótesis } & Beta estandarizado & Estadísticos t & P Valores & Decisión \\
\hline $\begin{array}{l}\text { (Actitud cognitiva }-> \\
\text { Actitud conductual) }\end{array}$ & 0,451 & 5,181 & 0 & Acepta h1 \\
VM -> DM & & & \\
$\begin{array}{l}\text { (Actitud afectiva -> } \\
\text { Actitud conductual) }\end{array}$ & 0,405 & 4,035 & 0 & Acepta h2 \\
AM -> DM & & & \\
\hline
\end{tabular}

Los resultados anteriores son coherentes con el planteamiento de Daems et al., (2019), puesto que se asemejan tanto en los antecedentes como en su orden de importancia; siendo primero el conocimiento que sobre un producto o una marca adquieren los consumidores para que, en un segundo momento desarrollen afectos hacia él o ella, y finalmente actúen privilegiando la racionalidad sobre la emotividad. También se puede afirmar que los datos aportan al estudio de las relaciones entre AM y DM indicadas por Burnasheva, et al. (2019), quienes sugieren la existencia de la relación positiva entre estas variables.

Finalmente, el coeficiente de determinación indica que la proporción de la variación total en la variable dependiente, explicada por las variables independientes del modelo, es de 0,677 , siendo este valor considerable para afirmar que el modelo y sus relaciones tienen capacidad explicativa de la defensa de una marca local, desde las actitudes cognitivas y afectivas.

\section{Conclusiones}

El objetivo de este estudio fue analizar las actitudes cognitivas y afectivas del consumidor en relación con la marca, con el fin de establecer cuál variable tiene mayor incidencia en su defensa. Los resultados demuestran que en las actitudes cognitivas se destaca la variable $C P$, puesto que los consumidores ven en La Catira una marca que ofrece productos de calidad con excelentes características; en virtud de ello, la marca les brinda confianza en sus decisiones de consumo.

Desde el análisis de la actitud afectiva, se infiere que es la dimensión con menor impacto en DM. Esto se debe a que una cifra cercana al $42 \%$ de las personas afirman no amar a La Catira, y aunque esto no significa que tengan un afecto negativo 
hacia la marca o que la odien, el análisis está orientado a la ausencia de un vínculo emocional fuerte entre el consumidor y La Catira. Esto demuestra que a pesar de tratarse de una marca local, todavía queda un camino por recorrer para alcanzar la categoría de marca amada, por la que se exprese una actitud afectiva.

De esta forma, se concluye que existe una incidencia de las actitudes cognitivas y afectivas de los consumidores en la defensa de una marca local; sin embargo, se perfilan como compradores que privilegian los aspectos de calidad del producto sobre los aspectos emocionales con la marca.

A nivel de implicaciones gerenciales, la defensa de la marca abordada desde la conducta del consumidor es un tema novedoso. Los resultados de la presente investigación demuestran que para la empresa analizada el incremento de los aspectos cognitivos representados en VM, junto con los aspectos afectivos de AM, permiten que los consumidores defiendan la marca local. Esto hace que el gerente comercial desarrolle estrategias de marketing no solamente con la finalidad de impulsar las ventas; también con el objetivo de incrementar la promoción desde la perspectiva del consumidor, como resultado de los altos niveles de confianza y afecto hacia la marca y la empresa en sí. Esta es una forma de orientación al mercado, donde los clientes se convierten en grandes aliados dispuestos a defender su marca ante el surgimiento de críticas o los pronunciamientos negativos de los detractores.

\section{Agradecimientos}

Los autores agradecen al Centro de Proyección Social de la Facultad de Ciencias Económicas, de la Universidad de los Llanos, por avalar mediante acta No. 09 del 8 de agosto de 2019, el curso libre: Fortalecimiento de la capacidad profesional en el diseño de estrategias de gestión de marca a partir de la investigación del consumidor, del que se desprende este manuscrito. También reconocen el apoyo obtenido del programa "Colombia
Científica y su componente Pasaporte a la Ciencia", en el marco del Foco Sociedad - Reto 2: Innovación social para el desarrollo económico y la inclusión productiva. Asimismo, agradecen a las personas que tomaron el curso mencionado, por su participación en la recolección de los datos.

\section{Referencias}

Aaker, D. (1996). Measuring brand equity across products and markets. California Management Review, 38 (3), 102-120. Recuperado de: https:// www.academia.edu/7284936/Aaker_1996_ Measuring_Brand_Equity_Across_Products_and_ Markets

Bagozzi, R. P., Batra, R., \& Ahuvia, A. (2017). Brand love: Development and validation of a practical scale. Marketing Letters, 28 (1), 1-14. doi: 10.1007/ s11002-016-9406-1

Batra, R., Ahuvia, A., \& Bagozzi, R. P. (2012). Brand love. Journal of Marketing, 76 (2), 1-16. doi:10.1509/ jm.09.0339

Burnasheva, R., Suh, Y. G., \& Villalobos, M. K. (2019). Sense of community and social identity effect on brand love: Based on the online communities of a luxury fashion brands. Journal of Global Fashion Marketing, 10 (1), 50-65. doi:10.1080/20932685.20 18.1558087

Carroll, B. A., \& Ahuvia, A. C. (2006). Some antecedents and outcomes of brand love. Marketing Letters, 17 (2), 79-89. doi:10.1007/ s11002-006-4219-2

Cavazos, A. J., \& Giuliani, A. C. (2008). El concepto de marketing bajo el paradigma relacional. Una agenda para Latinoamérica. Invenio, 11 (20), 41-52. Recuperado de: https://dialnet.unirioja.es/servlet/ articulo?codigo $=4225542$

Daems, K., De Pelsmacker, P., \& Moons, I. (2019). The effect of ad integration and interactivity on young 
teenagers' memory, brand attitude and personal data sharing. Computers in Human Behavior, 99, 245-259. doi: 10.1016/j.chb.2019.05.031

Dotson, J. P., Fan, R. R., Feit, E. M., Oldham, J. D., \& Yeh, Y. H. (2017). Brand attitudes and search engine queries. Journal of Interactive Marketing, 37, 105-116. doi: 10.1016/j.intmar.2016.10.002

Du, R. X., Joo, M., \&Wilbur, K. (2019). Advertising and brand attitudes: Evidence from 575 brands over five years. Quantitative Marketing and Economics, 17 (3), 257-323. doi: 10.1007/s11129-018-9204-6

Federación Nacional de Comerciantes (2019). FENALCO Meta pide declarar emergencia económica por problemas de la vía al llano. Recuperado de: http://www.fenalco.com.co/bienvenidos/ fenalco-meta-pide-declarar-emergencia-econ\%C3\%B3mica-por-problemas-de-la-v\%C3\%ADa-al-lla

Gill, M. S., \& Dawra, J. (2010). Evaluating Aaker's sources of brand equity and the mediating role of brand image. Journal of targeting, measurement and analysis for marketing, 18 (3/4), 189-198. doi: 10.1057/jt.2010.11

Keller, K. L. (1993). Conceptualizing, measuring, and managing customer-based brand equity. Journal of Marketing, 57 (1), 1-22. doi: https://doi. org/10.1177/002224299305700101

Lassar, W., Mittal, B., \& Sharma, A. (1995). Measuring customer-based brand equity. Journal of Consumer Marketing, 12 (4), 11-19. doi:10.1108/07363769510095270

Melancon, J. P., Noble, S. M., \& Noble, C. H. (2011). Managing rewards to enhance relational worth. Journal of the Academy of Marketing Science, 39 (3), 341-362. doi:10.1007/s11747-010-0206-5

Morales, J. F. (1999). Psicología Social. Madrid: Editorial McGraw-Hill.
Netemeyer, R. G., Krishnan, B., Pullig, C., Wang, G., Yagci, M., Dean, D., Ricks, J., \& Wirth, F. (2004). Developing and validating measures of facets of customer-based brand equity. Journal of Business Research, 57, 209-224. doi:10.1016/ S0148-2963(01)00303-4

Pappu, R., Quester, P. G., \& Cooksey, R. W. (2005). Consumer-based brand equity: improving the measurement - empirical evidence. Journal of Product \& Brand Management, 14 (3), 143-154. doi:10.1108/10610420510601012

Roy, S. K., Eshghi, A., \& Sarkar, A. (2013). Antecedents and consequences of brand love. Journal of Brand Management, 20 (4), 325-332. doi:10.1057/ bm.2012.24

Schmid, D. A., \& Huber, F. (2019). Brand love: Emotionality and development of its elements across the relationship lifecycle. Psychology \& Marketing, 36 (4), 305-320. doi:10.1002/mar.21180

Semana Rural (2018). La Vía al Llano: el incurable dolor de cabeza del Meta. Recuperado: de https:// semanarural.com/web/articulo/via-al-llano-cierre-de-la-carretera-bogota-villavicencio/641

Sürücü, O., Öztürk, Y. Okumus, F., \& Bilgihan, A. (2019). Brand awareness, image, physical quality and employee behavior as building blocks of customer-based brand equity: Consequences in the hotel context. Journal of Hospitality and Tourism Management, 40, 114-124. doi: 10.1016/j. jhtm.2019.07.002

Villarejo, A. F. (2002). Modelos multidimensionales para la medición del valor de marca. Investigaciones europeas de dirección y economía de la empresa, 8 (3), 12-44. Recuperado de: https://dialnet.unirioja. es/servlet/articulo?codigo $=634171$

Wallace, E., Buil, I., \& De Chernatony, L. (2014). Consumer engagement with self-expressive brands: Brand love and WOM outcomes. The 
Journal of Product and Brand Management, 23 (1), 33-42. doi: 10.1108/JPBM-06-2013-0326

Walz, A. M., \& Celuch, K. G. (2010). The effect of retailer communication on customer advocacy: the moderating role of trust. Journal of Consumer Satisfaction, Dissatisfaction \& Complaining Behavior, 23, 95-110. Recuperado de: https://jcsdcb. com/index.php/JCSDCB/article/view/25

Yoo, B., Donthu, N., \& Lee, S. (2000). An examination of selected marketing mix elements and brand equity. Academy of Marketing Science, 28 (2), 195-211. doi:10.1177/0092070300

Anexo 1. Instrumento de medida
Zarantonello, L., Formisano, M., \& Grappi, S. (2016). The relationship between brand love and actual brand performance: Evidence from an international study. International Marketing Review 33 (6), 806-824. doi: 10.1108/IMR-11-2015-0238

Zeithaml, V. (1988). Consumer perceptions of price, quality, and value: a means-end model and synthesis of the evidence. Journal of Marketing, 52, 2-22. doi: $10.2307 / 1251446$

\section{Anexos}

Por favor indique si está de acuerdo o no con las siguientes afirmaciones, relacionadas con su percepción sobre los productos alimenticios de La Catira, teniendo en cuenta que: muy en desacuerdo $=1$; en desacuerdo $=2 ;$ moderado acuerdo $=3 ;$ de acuerdo $=4$ y muy de acuerdo $=5$.

\begin{tabular}{|c|c|c|c|c|c|}
\hline Calidad & 1 & 2 & 3 & 4 & 5 \\
\hline \multicolumn{6}{|c|}{ La Catira ofrece productos de muy buena calidad } \\
\hline \multicolumn{6}{|c|}{ Los productos de La Catira son de confianza } \\
\hline \multicolumn{6}{|c|}{$\begin{array}{l}\text { Los productos de La Catira tienen excelentes } \\
\text { características }\end{array}$} \\
\hline \multicolumn{6}{|c|}{ Los productos de La Catira ofrecen buenos resultados } \\
\hline Notoriedad & 1 & 2 & 3 & 4 & 5 \\
\hline \multicolumn{6}{|c|}{ He oído hablar de la marca La Catira } \\
\hline \multicolumn{6}{|c|}{ Conozco la marca La Catira } \\
\hline \multicolumn{6}{|c|}{$\begin{array}{l}\text { Cuando pienso en productos lácteos, La Catira es } \\
\text { una de las marcas que me viene a la mente }\end{array}$} \\
\hline \multicolumn{6}{|c|}{$\begin{array}{l}\text { Puedo reconocer el símbolo o el logo de La Catira } \\
\text { frente a otras marcas competidoras de productos } \\
\text { lácteos }\end{array}$} \\
\hline Valor percibido & 1 & 2 & 3 & 4 & 5 \\
\hline \multicolumn{6}{|c|}{ La marca La Catira me ofrece lo que busco/necesito } \\
\hline \multicolumn{6}{|c|}{$\begin{array}{l}\text { La marca La Catira tiene buena relación calidad/ } \\
\text { precio }\end{array}$} \\
\hline Personalidad de marca & 1 & 2 & 3 & 4 & 5 \\
\hline
\end{tabular}




\begin{tabular}{|c|c|c|c|c|c|}
\hline Calidad & 1 & 2 & 3 & 4 & 5 \\
\hline \multicolumn{6}{|l|}{$\begin{array}{l}\text { Cuando uso la marca La Catira causo buena impre- } \\
\text { sión en los demás }\end{array}$} \\
\hline \multicolumn{6}{|l|}{$\begin{array}{l}\text { Tengo una imagen clara del tipo de persona que usa } \\
\text { la marca La Catira }\end{array}$} \\
\hline La empresa & 1 & 2 & 3 & 4 & 5 \\
\hline \multicolumn{6}{|l|}{ Confío en la empresa que fabrica los lácteos La Catira } \\
\hline \multicolumn{6}{|l|}{$\begin{array}{l}\text { La empresa que fabrica los lácteos La Catira es } \\
\text { admirable }\end{array}$} \\
\hline \multicolumn{6}{|l|}{$\begin{array}{l}\text { La empresa que fabrica los lácteos La Catira tiene } \\
\text { credibilidad }\end{array}$} \\
\hline Lealtad & 1 & 2 & 3 & 4 & 5 \\
\hline \multicolumn{6}{|l|}{ Me considero un consumidor leal a la marca La Catira } \\
\hline \multicolumn{6}{|l|}{$\begin{array}{l}\text { Cuando voy a comprar productos lácteos, la marca } \\
\text { La Catira es mi primera opción }\end{array}$} \\
\hline \multicolumn{6}{|l|}{$\begin{array}{l}\text { No compraría otras marcas si La Catira estuviera } \\
\text { disponible en la tienda, en el supermercado o en } \\
\text { cualquier otro punto de venta }\end{array}$} \\
\hline Amor a la marca & 1 & 2 & 3 & 4 & 5 \\
\hline \multicolumn{6}{|l|}{ Esta es una marca maravillosa } \\
\hline \multicolumn{6}{|l|}{ Esta marca me hace sentir bien } \\
\hline \multicolumn{6}{|l|}{ Esta marca me hace muy feliz } \\
\hline \multicolumn{6}{|l|}{ Amo esta marca } \\
\hline \multicolumn{6}{|l|}{ Esta marca es una delicia pura } \\
\hline \multicolumn{6}{|l|}{ Soy un apasionado de esta marca } \\
\hline \multicolumn{6}{|l|}{ Estoy muy apegado a esta marca } \\
\hline Defensa de la marca & 1 & 2 & 3 & 4 & 5 \\
\hline \multicolumn{6}{|l|}{$\begin{array}{l}\text { Rara vez pierdo la oportunidad de contarle a otros } \\
\text { cosas buenas sobre La Catira }\end{array}$} \\
\hline \multicolumn{6}{|l|}{$\begin{array}{l}\text { Intento que mis amigos y familiares compren los } \\
\text { productos de La Catira }\end{array}$} \\
\hline \multicolumn{6}{|l|}{$\begin{array}{l}\text { Llevaría a mis amigos/familiares a los puntos de } \\
\text { venta de La Catira porque creo que les gustaría estar } \\
\text { allí. }\end{array}$} \\
\hline $\begin{array}{l}\text { Defendería a La Catira si escuchara a alguien hablar } \\
\text { mal de ella }\end{array}$ & & & & & \\
\hline
\end{tabular}


Anexo 2. Fiabilidad y validez inicial de los constructos de primer orden

\begin{tabular}{|c|c|c|c|c|c|c|c|c|}
\hline Vari+A1:J33able & Dimensión & Ítem & $\begin{array}{l}\text { Cargas } \\
\text { iniciales }\end{array}$ & $\begin{array}{l}\text { Estadís- } \\
\text { tico t }\end{array}$ & $\begin{array}{l}\text { P } \\
\text { Valores }\end{array}$ & $\begin{array}{l}\text { Fiabilidad } \\
\text { compuesta }\end{array}$ & $\begin{array}{l}\text { Alfa } \\
\text { de } \\
\text { Cron- } \\
\text { bach }\end{array}$ & $\begin{array}{l}\text { Varianza } \\
\text { extraída } \\
\text { media } \\
\text { (AVE) }\end{array}$ \\
\hline \multirow[t]{20}{*}{ Actitud cognitiva (VM) } & $\mathrm{CP}$ & $\mathrm{CP} 1$ & 0,862 & 34,902 & 0 & 0,936 & 0,909 & 0,785 \\
\hline & & $\mathrm{CP} 2$ & 0,914 & 58,175 & 0 & & & \\
\hline & & $\mathrm{CP} 3$ & 0,895 & 50,329 & 0 & & & \\
\hline & & $\mathrm{CP} 4$ & 0,873 & 38,815 & 0 & & & \\
\hline & $\mathrm{N}$ & N1 & 0,798 & 22,972 & 0 & 0,88 & 0,819 & 0,647 \\
\hline & & N2 & 0,794 & 24,117 & 0 & & & \\
\hline & & N3 & 0,837 & 39,083 & 0 & & & \\
\hline & & N4 & 0,786 & 23,841 & 0 & & & \\
\hline & AS & AS1 & 0,854 & 61,483 & 0 & 0,912 & & \\
\hline & 0,856 & & & & & & & \\
\hline & 0,776 & & & & & & & \\
\hline & & AS2 & 0,848 & 41,678 & 0 & & & \\
\hline & & AS3 & 0,854 & 85,149 & 0 & & & \\
\hline & & AS4 & 0,798 & 60,095 & 0 & & & \\
\hline & & AS5 & 0,754 & 55,639 & 0 & & & \\
\hline & & AS6 & 0,803 & 44,613 & 0 & & & \\
\hline & & AS7 & 0,784 & 75,859 & 0 & & & \\
\hline & & AS8 & 0,749 & 64,402 & 0 & & & \\
\hline & L & L1 & 0,92 & 61,456 & 0 & 0,95 & 0,921 & 0,864 \\
\hline & & L2 & 0,953 & 143,435 & 0 & & & \\
\hline \multirow{10}{*}{$\begin{array}{l}\text { Actitud afectiva } \\
\text { (AM) }\end{array}$} & & L3 & 0,915 & 64,04 & 0 & 0,971 & 0,965 & 0,825 \\
\hline & & & & & & & & \\
\hline & & AM1 & 0,899 & 57,322 & 0 & & & \\
\hline & & AM2 & 0,903 & 67,678 & 0 & & & \\
\hline & & AM3 & 0,929 & 84,52 & 0 & & & \\
\hline & & & AM4 & 0,909 & 46,711 & 0 & & \\
\hline & & AM5 & 0,869 & 30,337 & 0 & & & \\
\hline & & DM2 & 0,908 & 55,025 & 0 & & & \\
\hline & & DM3 & 0,849 & 33,1 & 0 & & & \\
\hline & & DM4 & 0,897 & 52,344 & 0 & & & \\
\hline
\end{tabular}


Anexo 3. Fiabilidad y validez final de los constructos de segundo orden

\begin{tabular}{|c|c|c|c|c|c|c|c|}
\hline Variable & Ítem & $\begin{array}{l}\text { Cargas } \\
\text { finales }\end{array}$ & $\begin{array}{c}\text { Estadístico } \\
t\end{array}$ & P Value & $\begin{array}{l}\text { Fiabilidad } \\
\text { compuesta }\end{array}$ & $\begin{array}{l}\text { Alfa de } \\
\text { Cronbach }\end{array}$ & $\begin{array}{c}\text { Varianza } \\
\text { extraída } \\
\text { media } \\
\text { (AVE) }\end{array}$ \\
\hline \multirow{4}{*}{$\begin{array}{l}\text { Actitud } \\
\text { cognitiva } \\
\text { (VM) }\end{array}$} & $\mathrm{CP}$ & 0,834 & 36,478 & 0 & \multirow{4}{*}{0,928} & \multirow{4}{*}{0,897} & \multirow{4}{*}{0,765} \\
\hline & $\mathrm{N}$ & 0,856 & 34,806 & 0 & & & \\
\hline & AS & 0,951 & 143,586 & 0 & & & \\
\hline & L & 0,852 & 49,861 & 0 & & & \\
\hline $\begin{array}{l}\text { Actitud } \\
\text { afectiva }\end{array}$ & AM1 & 0,899 & 56,057 & 0 & \multirow{7}{*}{0,971} & \multirow{7}{*}{0,965} & \multirow{7}{*}{0,825} \\
\hline \multirow[t]{6}{*}{ (AM) } & AM2 & 0,903 & 64,347 & 0 & & & \\
\hline & AM3 & 0,929 & 83,891 & 0 & & & \\
\hline & AM4 & 0,909 & 46,358 & 0 & & & \\
\hline & AM5 & 0,869 & 31,173 & 0 & & & \\
\hline & AM6 & 0,933 & 88,484 & 0 & & & \\
\hline & AM7 & 0,914 & 66,52 & 0 & & & \\
\hline $\begin{array}{l}\text { Actitud } \\
\text { conductual }\end{array}$ & DM1 & 0,919 & 65,851 & 0 & \multirow{4}{*}{0,941} & \multirow{4}{*}{0,916} & \multirow{4}{*}{0,799} \\
\hline \multirow[t]{3}{*}{ (DM) } & DM2 & 0,908 & 54,811 & 0 & & & \\
\hline & DM3 & 0,849 & 33,303 & 0 & & & \\
\hline & DM4 & 0,896 & 52,596 & 0 & & & \\
\hline
\end{tabular}

